\title{
A Saliency Detection Method Based on Global Contrast
}

\author{
Chun-yan $\mathrm{Yu}^{1,2}$, Wei-shi Zhang ${ }^{1}$ and Chun-li Wang ${ }^{1}$ \\ ${ }^{1}$ Institute of Information and Science Technology, Dalian Maritime University \\ ${ }^{2}$ Key Laboratory of Intelligent Computing \& Signal Processing, Ministry of \\ Education, Anhui University, No.3 Feixi Road, Hefei \\ yuchunyan1997@126.com,teesiv@163.com,clwang@dlmu.edu.cn
}

\begin{abstract}
To highlight the saliency object clearly from the foreground, we propose a saliency detection method based on global contrast with cluster. Due to the fact that background pixels usually have similar patches, we use cluster analysis to merge the background regions. By using mean shift filter, the background pixels with similar color level are clustered and the saliency calculation can be decreased a lot. In the method, we use the contrast of color feature with all the other pixels to compute the saliency map. A weight coefficient is utilized to improve the detection accuracy in global contrast differences evaluation. The results of extensive experiments on public dataset show that our method perform well and can highlight the salient object clearly against the other five state-of-the-art methods. Besides, we demonstrate that the applications in image segmentation and fusion with our saliency map can get satisfactory results.
\end{abstract}

Keywords: Saliency detection, mean shift filter, saliency map, global contrast

\section{Introduction}

The aim of visual attention demonstrated that human perception of an image is developed by a combination of attention, eye movements, and memory [1]. Visual Attention acts as a gatekeeper, passing a relevant subset of the stimuli to one or more limited capacity processes [2]. In the front of a complex scene, attention enables object recognition processes to work on a single item at a time. In this way, humanity can only attend to a single thing at a time, and visual attention guilds people to some "salient" regions. The main task of saliency detection is to detect regions or objects of interested in images. In recent years, saliency detection has been used widely in computer vision, including object recognition [3-5], image compression [6, 7], image matching [8,9], and image segmentation [10-13].

The existing saliency detection models based on psychology can be categorized into two classes: bottom-up and top-down models. On one hand, attention can be driven exogenously, by an external stimulus event that automatically draws attention to a particular location [14]. The bottom-up model [15-19] following the exogenous attention is referred to as "stimulus-driven", and the attention factors is related to the characteristics of the scene, including color, contrast, and orientation, and saliency maps show pixels which are related to low level properties in the initial visual scanning process. On the other hand, in endogenous cueing, attention is presumed to be under the overt control of the subject. The top-down model [20-23] following endogenous attention is a goal-driven attention model which is closely related to specific cognitive task, it needs to analysis the high-level semantic information with prior knowledge to get the saliency region.

A number of saliency analysis methods and saliency detection models based on different mathematics theory have been proposed. Based on Koch \& Ullman visual attention model, the Itti model [24] is the most classic bottom-up visual saliency computational model, and a lot of bottom-up and top-down models are original from it. In 
the model, pre-attentive multi-scale features (color, intensity, orientation) extraction were decomposed from the image firstly, then feature maps are computed with center-surround operation in each of those feature channels, and finally, the saliency map is composed with the feature maps competition. Different from Itti model, Hou et al. [25] presented a simple method for the visual saliency detection. Given an input image, the spectral residual defined as the difference between the log spectrum and the averaged spectrum denotes the saliency information of the image, and the author also proposed a fast method to construct the corresponding saliency map in spatial domain. In [16], supported by the four psychological evidences, the author proposed a novel algorithm for context-aware saliency detection. Except for the dominant objects, the saliency region by the CA algorithm also includes the unique parts of the background. Furthermore, to enhance the saliency detection, a single-scale local-global saliency operator is used to get the saliency region, The method used multiple scales and weighted prior-knowledge as coefficient. In [17], using image color statistics, a histogram-based contrast (HC) method was proposed to define saliency values for image pixels. The $\mathrm{HC}$ algorithm evaluates global contrast differences of the image, and the saliency of a pixel is defined as color contrast to all other pixels. In the same paper, utilizing spatial coherence of the image, a region-based contrast (RC) method was also proposed to compute the saliency region.

The ideal saliency map should ignore all the background, and emphasize the dominant object only. However, for image with complex background, the saliency maps in the above methods highlight the background more or less. In this paper, we hope to detect the salient objects separated from the background part. To achieve this goal, based on the famous HC algorithm, we propose a bottom-up saliency detection method which utilizes mean shift cluster and global contrast computation. Compared with the current state-of-art saliency detection models, our method has great improvement on the salient objects highlight. The experimental results on public data sets show that our method outperforms other saliency methods, and has saliency detection robustness even for images with complex background.

The remainder of the paper is organized as follows: in Section 2, we briefly review the HC saliency detect algorithm; in Section 3, we present the proposed method and experimental results are presented in Section 4, we demonstrate two applications with our saliency map in Section 5 and conclusions are drawn in Section 6.

\section{HC Algorithm}

In [17], Cheng et al. proposed the histogram-based contrast (HC) saliency detection method. It is bottom-up data driven saliency detection using global contrast of image, and the principles which the author complied with are listed as following:

(1) Saliency detection method should separate a large-scale object from its surroundings

(2) Considering global contrast, the similar image regions can be assigned a comparable saliency values.

(3) In saliency computation, the nearby regions play more importance than the distant regions.

(4) The saliency detection function should be completed fast and easily.

In $\mathrm{HC}$ algorithm, for an image $I$, the saliency of a pixel $U_{n}$ is defined as

$$
S\left(I_{n}\right)=\sum_{\forall I_{n} \in I} D\left(I_{j}, I_{n}\right)
$$

Further, the definition can be modified as:

$$
S\left(I_{k}\right)=S\left(c_{l}\right)=\sum_{j=1}^{n} f_{j} D\left(c_{l}, c_{j}\right)
$$


Where, $c_{l}$ is the color value of pixel $I_{k}, n$ is the pixel colors number, and $f_{j}$ is the probability of pixel color $c_{j}$.

In the computation, to improve the running time, the authors utilized color quantization method to reduce the number of colors. And to reduce the noisy saliency value [17], the saliency value of each color was replaced by the weighted average saliency values of similar colors. The definition of saliency map is as follows:

$$
S^{\prime}(c)=\frac{1}{(m-1) T} \sum_{i=1}^{m}\left(T-D\left(c, c_{i}\right)\right) S\left(c_{i}\right)
$$

Where $T=\sum_{i=1}^{m} D\left(c, c_{i}\right)$ is the sum of distances between $\operatorname{color} c_{i}, m$ is the nearest pixels, generally, it is set to 4 in [17].

\section{The Proposed Method}

\subsection{Mean Shift Filter}

Color feature analysis is a low-level digital image processing and image understanding method. Each data in the image can be mapped to a multi-dimensional feature vector color. Because of the probability density function of the image is unknown, a common practice is to make assumptions about the probability density function, so that cluster analysis [26] is the most appropriate method of feature space analysis, each cluster distribution is assumed to obey the Gaussian mixture distribution, usually Gaussian mixture distribution parameters can be estimated by minimizing the error criterion. The most commonly used non-parametric estimation method is based on kernel density estimation which uses the kernel function $K(x)$, for each sample $x_{i}$, a positive definite matrix is utilized to represent the relative scale and the shape of the window of $x_{i}$.

In this paper, we utilize the traditional mean-shift algorithm to cluster the original image before detected. The mean shift algorithm was originally presented in 1975 by Fukunaga and Hostetler [27], and is developed by [28] [29]. Nowadays, it has been widely applied in computer vision and image processing area. In application with image filter, it is used as a type of nonparametric clustering method. Given $n$ data points $x_{i}, i \in[1, n]$, in the space $R^{d}$, in the mean-shift theory, the kernel density gradient estimation of $x$ is defined as

$$
\nabla P(x)=\frac{1}{n} \sum_{i=1}^{n} \nabla K\left(x-x_{i}\right)
$$

Using the Kernel form

$$
K\left(\mathbf{x}-\mathbf{x}_{i}\right)=c k\left(\left\|\frac{\mathbf{x}-\mathbf{x}_{i}}{h}\right\|^{2}\right)
$$

Where $h$ is the size of window, $c$ is normalization constant. The kernel function is usually defined by

$$
K(\mathbf{x})=e^{-\frac{\|x\|}{2 \sigma^{2}}}
$$

Denoted by

$$
g(x)=-K^{\prime}(\mathbf{x})
$$

We get 


$$
\nabla P(\mathbf{x})=\frac{c}{n} \sum_{i=1}^{n} \nabla k_{i}=\frac{c}{n}\left[\sum_{i=1}^{n} g_{i}\right]\left[\frac{\sum_{i=1}^{n} \mathbf{x}_{i} g_{i}}{\sum_{i=1}^{n} g_{i}}-\mathbf{x}\right]
$$

The mean shift vector can be computed by

$$
m(x)=\left[\frac{\sum_{i=1}^{n} x_{i} g\left(\frac{\left\|x-x_{i}\right\|^{2}}{h}\right)}{\sum_{i=1}^{n} g\left(\frac{\left\|x-x_{i}\right\|^{2}}{h}\right)}-x\right]
$$

To decrease the numbers of color, we only use the color level domain. Due to the fact that Luv and Lab color space are more compatible with human perception than RGB space, in this paper, the vector $x$ is defined as: $x=\left(I_{l u v}\right)$.

Using the weighted average of vector $x$ as feature, we compute the mean shift vector by the following equations:

$$
\begin{array}{r}
\nabla P(I)=\frac{1}{n} \sum_{i=1}^{n} \nabla K\left(\sqrt{\left(l-l_{i}\right)^{2}+\left(u-u_{i}\right)^{2}+\left(v-v_{i}\right)^{2}}\right) \\
m(I)=\left[\frac{\sum_{i=1}^{n}\left(I_{i}\right) g\left(\frac{\left\|\sqrt{\left(l-l_{i}\right)^{2}+\left(u-u_{i}\right)^{2}+\left(v-v_{i}\right)^{2}}\right\|^{2}}{h}\right)}{\sum_{i=1}^{n} g\left(\frac{\left\|\sqrt{\left(l-l_{i}\right)^{2}+\left(u-u_{i}\right)^{2}+\left(v-v_{i}\right)^{2}}\right\|^{2}}{h}\right)}-I\right]
\end{array}
$$

Given initial $x_{i}, g(x)$ and $h, \varepsilon$ denotes the error, the mean shift filter algorithm procedure can be described as:

(1) Compute $\mathrm{m}(\mathrm{x})$ by the equation (11).

(2) Let $x_{i}=m(x)$.

(3) If ( $\|m(x)-x\|<\varepsilon$ ), end loop; else go to step (1).

The performance of the mean shift filter is shown in this section. In this experiment, we apply the filter to four images with complex background. The first row of Figure 1 list the original images and the processed images after mean shit filter are list on the second row. Objectively, as shown in Figure 2, we list all the color distribution of the above images. The upper row of Figure 2 are the color distribution of original images and the color distribution of corresponding images after filter are shown in the under row. Specifically, the comparison of numbers of color is shown in the Figure 3. It is clearly seen that quite a few colors are eliminated after the mean shift filtering. Furthermore, the reduction percentages are list in Table 1. It can be observed that $15 \%$ colors are reduced at least, and the average reduction percentage is $22.8 \%$.
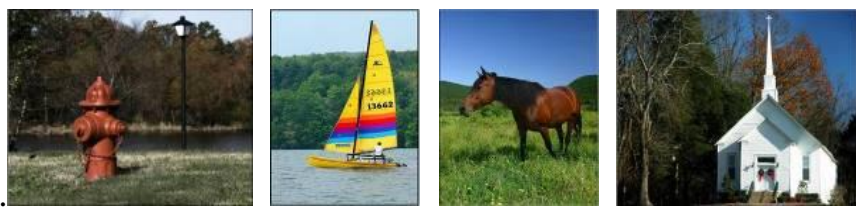

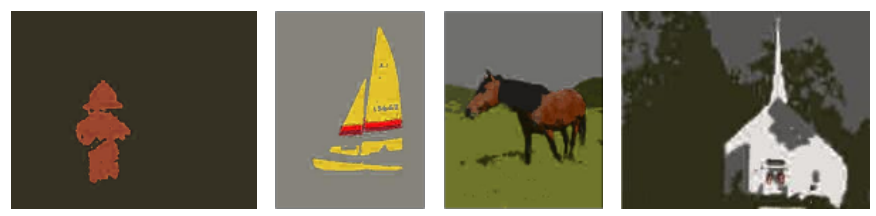

Figure 1. Performance of Mean Shift Filter
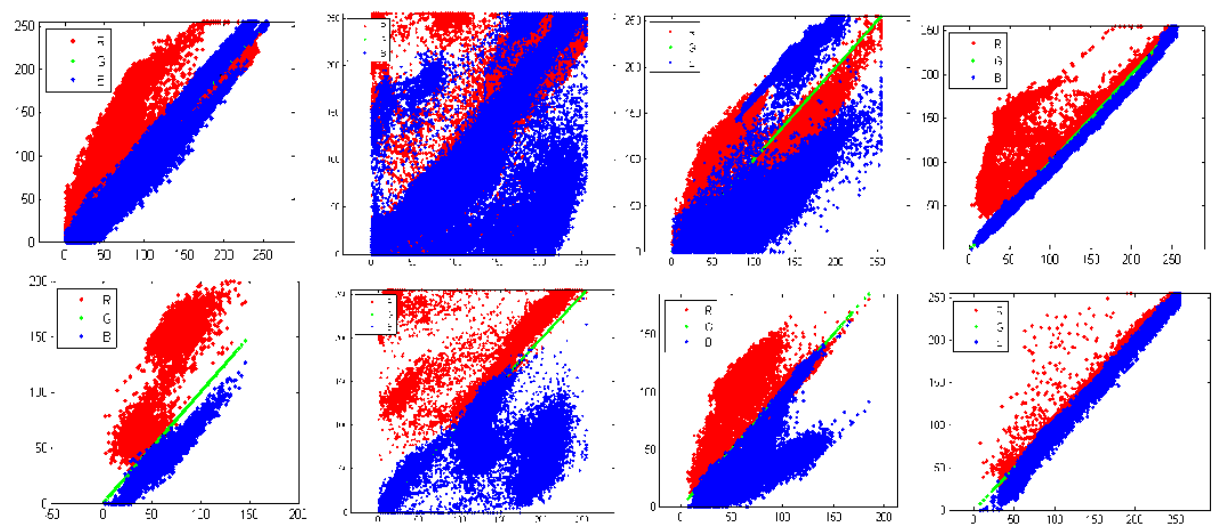

Figure 2. Color Distribution of Images in Figure 1
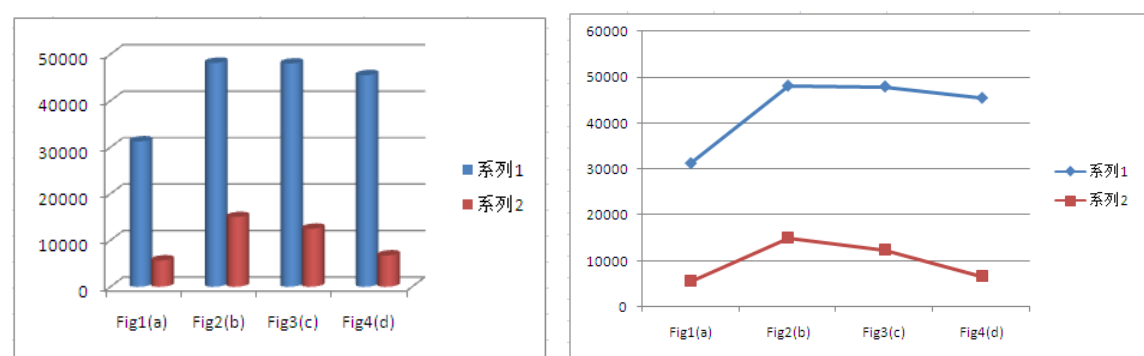

Figure 3. Histogram and Line Char of Color Numbers of Images in Figure 1

Table 1. Comparison of Color Numbers of Images in Figure 1

\begin{tabular}{|c|c|c|c|}
\hline Fig.1(a) & 31250 & 5657 & $18.1 \%$ \\
\hline Fig.1(b) & 48153 & 14985 & $31.1 \%$ \\
\hline Fig.1(c) & 47983 & 12467 & $26.0 \%$ \\
\hline Fig.1(d) & 45493 & 6728 & $14.8 \%$ \\
\hline
\end{tabular}

\subsection{Saliency Map Computation}

In this paper, due to the fact that the vision system is sensitive to contrast in visual signal, we compute the values of saliency map by using color statistics of the clustered image. Specifically, the saliency of a pixel is defined by the color contrast to all other pixels. Meanwhile, in order to improve the accuracy of the saliency map computation, we define the weight coefficient $w\left(I_{i}\right)$ to emphasize the effect of every pixel, which means the weight of the pixel $I_{i}$. The difference between the original image and the processed image denotes as

$$
\Delta\left(I_{i}\right)=\left|I_{i}-I(m)_{i}\right|
$$

As we know, the bigger $\Delta\left(I_{i}\right)$ means the less saliency. In this paper, the variance rate $\eta_{i}$ of pixel $I_{i}$ is defined as 


$$
\eta_{i}=\frac{\Delta\left(I_{i}\right)}{I_{i}}
$$

Accordingly, the weight $w\left(I_{i}\right) \in[0,1]$ is defined by

$$
w\left(I_{i}\right)=\frac{1}{1+\lambda \eta_{i}^{2}}
$$

In implementation, to reduce the number of colors further, $R, G, B$ in three channels is divided into 16 intervals. In this way, the colors change into $16 \times 16 \times 16$, normalized to the range [0:1]. Because of the visual system is more sensitive to green color, the definition of color quantization denotes as

$$
(r, g, b)=r+256 \times g+16 \times b
$$

Further, the saliency map of image $I$ is defined as:

$$
S\left(C_{l i}\right)=\sum_{p=1}^{n}\left\|C_{l i}, C_{l p}\right\| \cdot w\left(C_{l i}\right)
$$

Where $C_{l i}$ is the color value of pixel $I(i), n$ is the number of distinct pixel colors, and $w\left(C_{l i}\right)$ is the weight coefficient of pixel color $C_{l i},\left\|C_{l i}, C_{l p}\right\|$ is the Euclidean distance between the two patches in color space.

Note that the same color level pixels have the same saliency value by the definition, the equation (16) can be written as:

$$
S\left(I(m)_{i}\right)=S\left(C_{l i}\right)=\sum_{j=1}^{k} \frac{n_{j}}{k}\left\|C(m)_{l i}, C(m)_{l j}\right\| \cdot \frac{1}{1+\lambda \eta_{i}^{2}}
$$

Where, $c_{l}$ is the color value of pixel $I_{i}, k$ is the pixel colors number, and $n_{j}$ is the probability of pixel color $C_{l j}$. Due to the fact that the perceived difference approximates Euclidean distance in CIE L*a*b color space, in this procedure, the CIE L*a*b color space is used.

In HC, in order to reduce noisy saliency results caused by using similar colors, a color space smoothing step is used to refine the randomness value for each color. The author computed the saliency value by the weighted average saliency values of similar colors. In our method, we utilize mean shift filter to decrease colors a lot, it can efficiently compute color contrast without the drawback caused by similar color estimation, in this way, our method can detect the saliency region separately from the background, and the computation time of saliency map is faster than the HC algorithm.

Accordingly, the procedure of our proposed method is described as follows:

(1) with given the value of $h$, process the original image by mean shift filter to get a new $I(m)$.

(2) compute weight coefficient of all the pixels by equation (114).

(3) compute the saliency map by equation (17).

\section{Experimental Results}

In this section, we evaluated our saliency detection method and presented the comparison with several state-of-the-art models on available dataset provided by Achanta [33] et al. All the experiments run on a computer with Intel Celeron processor M $2.7 \mathrm{GHz}$, 4GB memory, the OS is Microsoft Windows XP, and the running plat is MATLAB $2012 b$.

The dataset which is publicly available contains 28000 natural images with has the human-marked labeled ground truth for salient objects. We compute saliency maps with all the methods for 1000 images in the database. We compare the proposed method with 
five state-of-the-art methods: Itti [24], gvbs [18], FC [19], CA [16], and HC [17]. For fair comparison, we use the authors' codes. In Figure 4, it is observed that the salient objects are well detected with our method, and the saliency region uniformly highlighted the foreground objects with continuous boundaries. Figure.5 shows all the saliency regions with all the methods and the ground truth images provided by the dataset, in spite of the complex background, the given result images show that our method can outstand foreground. It can be seen that for the given ground truth, our method achieved an improvement over the other methods.
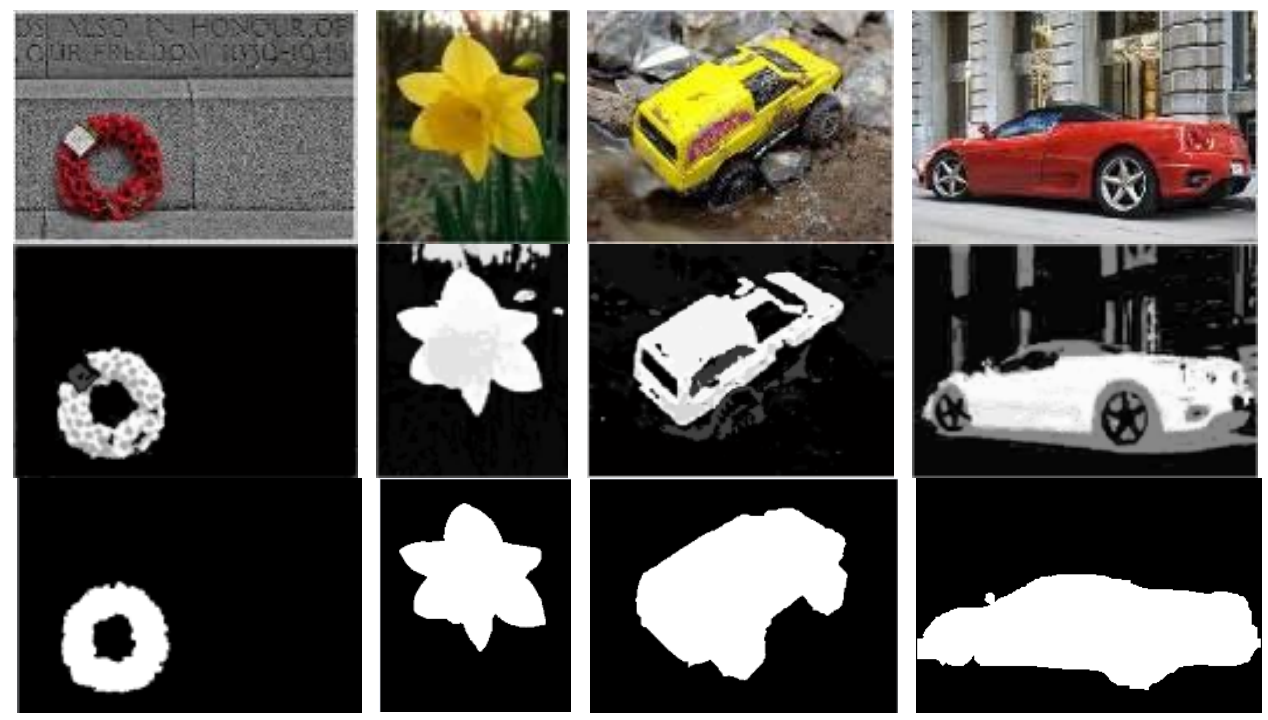

Figure 4. Saliency Object Detection by Our Method

In order to obtain a quantitative evaluation, we compute the precision and recall values of all the approaches respectively. To plot the pr curves, we need to binarize the saliency maps, in our experiments, we set the threshold value within range from 0 to 255 . Same as prior approaches, the values of precision and recall are defined by

$$
p_{r e}=\frac{\left|s_{g} \cap s_{f}\right|}{s_{f}}, r_{e c}=\frac{\left|s_{g} \cap s_{f}\right|}{s_{g}}
$$

Where $s_{f}$ denotes the pixels of the binary saliency regions foreground. $s_{g}$ denotes the pixels of the ground truth foreground.

The precision-recall curve is used to evaluate the performance of salient object location. In this experiment, we use 1000 publicly available benchmark images, and the bandwidth matrix $h$ for the color range scales of our method is set as 10 . The other state-of-art methods use their original codes. In many occasions, high precision and high recall are both needed. Figure 6 shows all the precision-recall curves of these methods. It is clearly observed that our method outperform the other six methods, and it can eliminate more background and outstand the foreground images. Our method achieves the highest precision, higher recall. Overall, our proposed saliency detection method achieves higher performance than the other method mentioned above. 


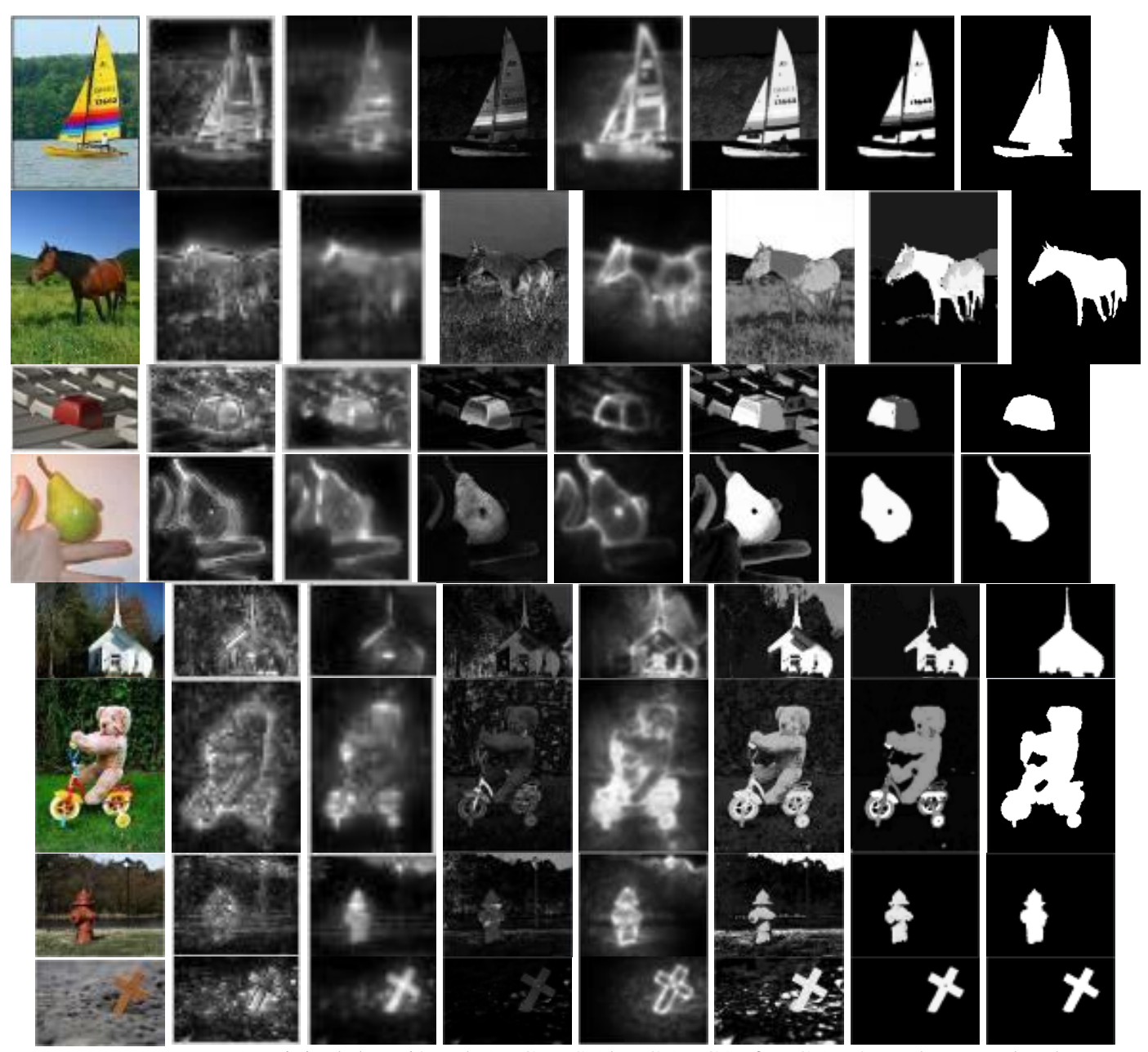

(a)original (b) Itti/Koch (c) GVBS (d) FC (e) CA (f) HC (g) Ours (h) groundtruth

Figure 5. Visual Comparison of Saliency Maps

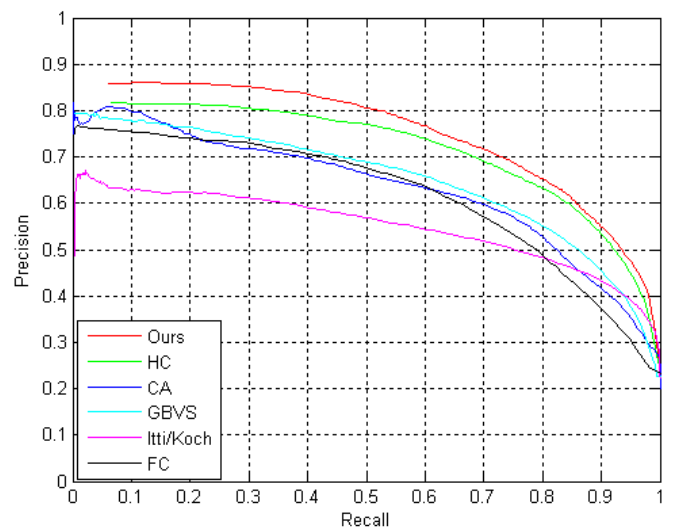

Figure 6. Comparison of Precision-recall Curves of Saliency Maps

\section{Applications}

\subsection{Image Segmentation}

Image segmentation is one of the most important basic pre-processing in many fields, the main task of segmentation is suggested to identify the salient regions in the images 
Active contour model with level set has been widely used in recent years. Among all the region-based models relied on the level set method, the Chan-Vese (CV) model [30] is the most classic and popular one. Using the saliency map for original image with initial evolution curve, the R-DRLSE model based on CV model [31] can detect the object easily. The experimental results are presented in the Figure 7. With the same initial curve shown in Figure 7(b), the result of saliency map with R-DRLSE model is shown in Figure 7(c), Figure 7(e) shows the segmentation result of the original image with CV model, it can be observed that the desired object is not detected successfully. The corresponding final level set function with the zero level set in red solid line as shown in Figure 7(f) and Figure 7(g).

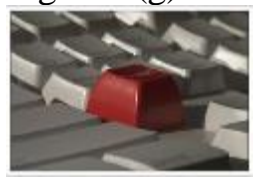

(a)

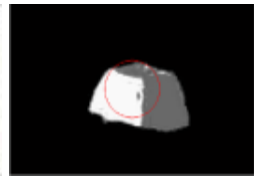

(b)

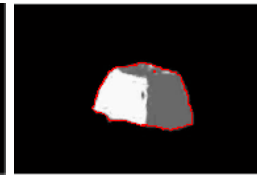

(c)

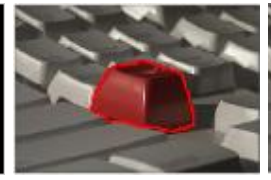

(d)

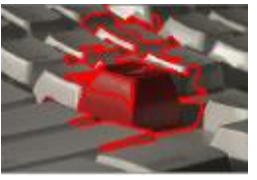

(e)

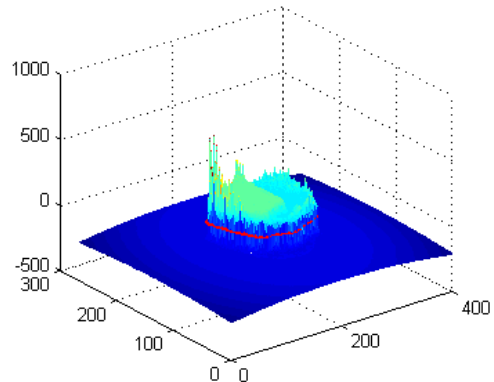

(f)

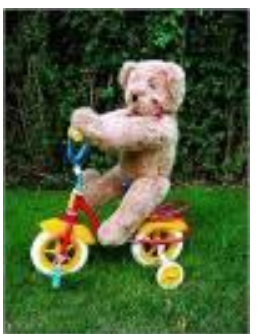

(h)

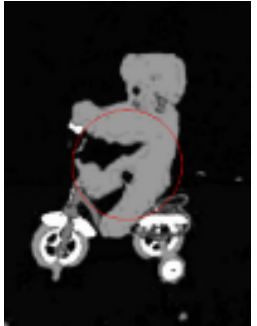

(i)

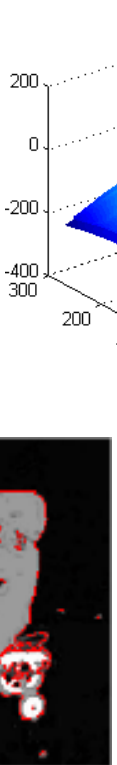

(j)

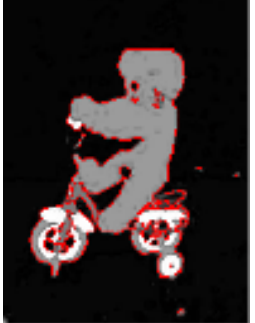

(g)

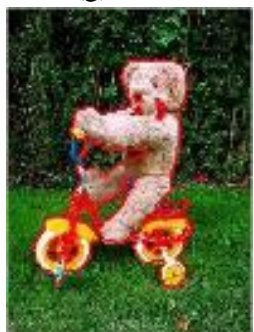

(k)

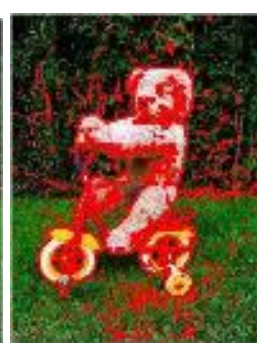

(1)

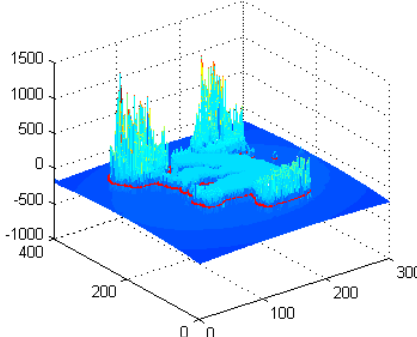

(m)

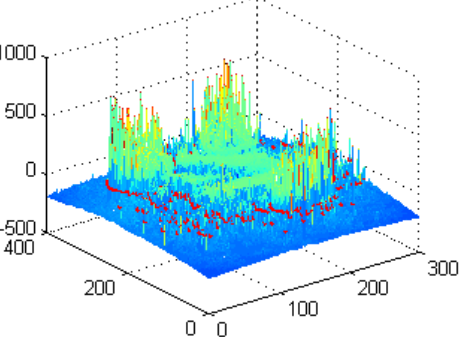

(n)

Figure 7. CV Model Segmentation Results with Our Saliency Map

\subsection{Image Fusion}

The best known Laplacian pyramid has been widely utilized as a useful multiple-scale tool for a variety of image processing applications, in this paper, we implement the Laplacian pyramid image fusion [32] with our saliency map. The basic procedure of Laplacian pyramid fusion is described as: firstly, perform a pyramid decomposition on the sources images, then integrated with decompositions to a composite representation, lastly, the fused image is reconstructed by performing an inverse pyramid transform., In the implementation, we substitute saliency map for the region mask in original Laplacian pyramid to create the blended image. The image fusion results images is shown in Figure 
8. The left two columns show the two input images, by using the saliency map as region mask, the blended images result are listed in the last columns. It can be observed that with our saliency map, the salient object of the first source image is blended well with the second source image.

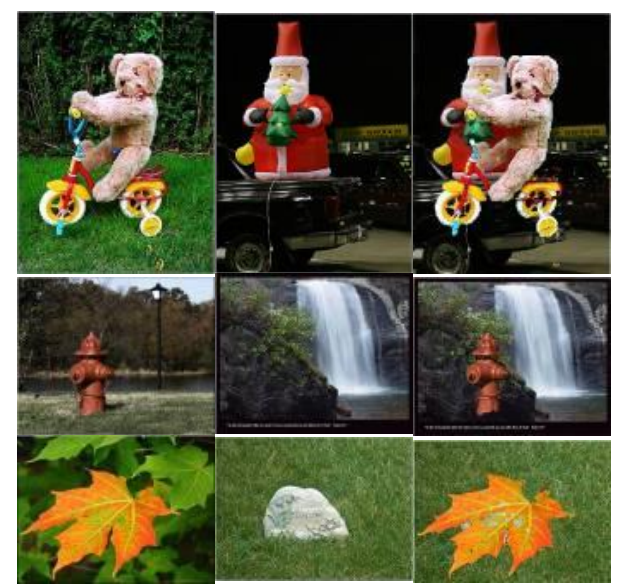

Figure 8. Laplacian Pyramid Blend of Two Images of Saliency Map

\section{Conclusion}

In this paper, we propose a bottom-up saliency detection method based on global contrast. Using the mean shift filter, the original image can decrease a few colors, which is useful to the saliency map computation. Besides, we use the weight coefficient to improve the saliency detection accuracy. Experimental results demonstrate the effectiveness of our filtering method, and the saliency map with our method highlights the salient object more clearly than the other state-of-the-art methods mentioned above. Furthermore, our method has the higher precision and recall too. In addition, we provide the contribution of our method with two applications: image segmentation with level set method and image fusion.

\section{Acknowledgments}

The authors would like to thank all the reviewers for their constructive comments. This research was supported by National Natural Science Foundation of China (No. 61370070), the Fundamental Research Funds for the Central Universities (Grant No. 3132013039 and 3132013040), Open Research Fund by Key Laboratory of Intelligent Computing \& Signal Processing, Ministry of Education, (Program 2014-004).

\section{References}

[1] K. R. Kluender and D. M. Levi, "Sensation and Perception”, Sinauer Associates, Incorporated, (2012).

[2] J. Wolfe, "Visual attention. In: De Valois KK", San Diego, CA: Academic Press; (2000), pp. 335-386.

[3] A. Shokoufandeh, I. Marsic and S. J. Dickinson, "View-based object recognition using saliency maps", Image and Vision Computing, vol. 17, no 1, (1999), pp. 445-460.

[4] Z. Ren, S. Gao, Liang-Tien and I. W. Tsang, "Region-based Saliency Detection and Its Application in Object Recognition", IEEE Transactions on Circuits and Systems for Video Technology, vol. 24, no 5, (2013), pp. 769 -779.

[5] S. Frintrop, A. Nuchter, H. Surmann and J. Hertzberg, "Saliency-based object recognition in 3D data", 2004 IEEE/RSJ International Conference on Intelligent Robots and Systems, vol. 3, (2004), pp. 21672172.

[6] Y. Fang, Z. Chen, W. Lin and Lin C.-W., "Saliency Detection in the Compressed Domain for Adaptive Image Retargeting", IEEE Transactions on Image Processing, vol. 21, no. 9, (2012), pp. 3888-3901. 
[7] F. Zund, Y. Pritch, A. Sorkine-Hornung, S. Mangold and T. Gross, "Content-aware compression using saliency-driven image retargeting”, IEEE International Conference on Image Processing (ICIP), (2013), pp. 1845-1849.

[8] A. Toshev, J. Shi and K. Daniilidis, "Image Matching via Saliency Region Correspondences", IEEE Conference on Computer Vision and Pattern Recognition, (2007), pp. 1-8.

[9] L. Li, S. Jiang, Z.-J. Zha, Z. Wu and Q. Huang, "Partial-Duplicate Image Retrieval via Saliency-Guided Visual Matching”, IEEE MultiMedia, vol. 20 , no. 3, (2013), pp. 13- 23.

[10] Q. Li, Y. Zhou and J. Yang, "Saliency based image segmentation", International Conference on Multimedia Technology (ICMT), (2011), pp. 5068-5071.

[11] T. A. Ta, T. H. Cao and T. V. Nguyen, "The Hybrid Approach of Image Segmentation Using MeanShift and Saliency Maps", International Conference on Knowledge and Systems Engineering (KSE), (2012), pp. $11-17$.

[12] J. Zhao, S. Chen, D. Zhao, H. Zhu and X. Chen, "Unsupervised saliency detection and a-contrario based segmentation for satellite images", International Conference on Sensing Technology (ICST), (2013), pp. 678- 681.

[13] R. Pal, P. Mitra and J. Mukhopadhyay, "Generation of groundtruth data for visual saliency experiments using image segmentation”, International Congress on Image and Signal Processing (CISP), (2012), pp. 889-893.

[14] M. M. Chun and J. M. Wolfe, "Visual Attention. In B. Goldstein", Blackwell Handbook of Perception Oxford, UK: Blackwell Publishers Ltd, (2001), pp. 272-310.

[15] A. Toet, "Computational versus Psychophysical Bottom-Up Image Saliency: A Comparative Evaluation Study", IEEE Transactions on Pattern Analysis and Machine Intelligence, vol. 33, no 11, (2011), pp. 2131-2146.

[16] S. Goferman, L. Zelnik-Manor, and A. Tal, "Context-aware saliency detection”, In CVPR, (2010).

[17] M.-M. Cheng, G.-X. Zhang, N. J. Mitra, X. Huang and S.-M. Hu, "Global Contrast based Salient Region Detection", IEEE CVPR, (2011), pp. 409-416.

[18] J. Harel, C. Koch, and P. Perona, "Graph-based visual saliency", Advances in neural information processing systems, (2007), pp. 545-552.

[19] R. Achanta, S. Hemami, F. Estrada and S. Süsstrunk, "Frequency-tuned Salient Region Detection”, IEEE International Conference on Computer Vision and Pattern Recognition (2009) , pp. 1597-1604.

[20] Y. Hua, Z. Zhao, H. Tian, X. Guo and A. Cai, "A probabilistic saliency model with memory-guided top-down cues for free-viewing", IEEE International Conference on Multimedia and Expo (ICME), (2013), pp. 1-6.

[21] J. Yang and M.-H. Yang, "Top-down visual saliency via joint CRF and dictionary learning", IEEE Conference on Computer Vision and Pattern Recognition (CVPR), (2012), pp. 2296 -2303.

[22] D. Kang, S. Lee and Y.-B. Lee, "Human visual attention with context-specific top-down saliency", IEEE International Conference on Robotics and Biomimetics (ROBIO), (2011), pp. 2055-2060.

[23] F. Barranco, J. Diaz, E. Ros and B. Pino, "Real-Time Visual Saliency Architecture for FPGA with Top-Down Attention Modulation", IEEE Transactions on Industrial Informatics, vol. 10, no. 3, (2014), pp. 1726-1735.

[24] L. Itti, C. Koch, and E. Niebur. "A Model of Saliency-BasedVisual Attention for Rapid Scene Analysis", PAMI, (1998), pp. 1254-1259.

[25] X. Hou and L. Zhang, "Saliency detection: A spectral residual approach", In CVPR, (2007), pp. 1-8.

[26] Q. Guo, X. Chang and H. Chu, "Clustering Analysis Based on the Mean Shift", International Conference on Mechatronics and Automation, (2007), pp. 309-313.

[27] K. Fukunaga and L. D. Hhstetler, "The Estimation of the Gradient of a Density Function, with Applications in Pattern Recognition", IEEE Transactions on information theory, vol. 21, no. 1, (1975), pp. 32-40.

[28] Y. Cheng, "Mean Shift Mode Seeking, and Clustering", IEEE Transactions on pattern analysis and machine intelligence, vol 17, no 8, (1995), pp. 790-799.

[29] D. Comaniciu and P. Meer, "Mean Shift: A Robust Approach toward Feature Space Analysis", IEEE transaction on PAMI, vol. 24, no. 5. (2002), pp. 603-619.

[30] T. Chan and L. Vese, "Active contours without edges", IEEE Trans. Image Process, vol. 10, no. 2, (2001), pp. 266-277.

[31] C.-y. Yu, W.-s. Zhang, Y.-y. Yu and Y. Li, "A novel active contour model for image segmentation using distance regularization term", Computers and Mathematics with Applications, vol. 65, no. 11, (2013), pp. 1746-1759.

[32] P. J. Burt and E. H. Adelson, "The Laplacian pyramid as a compact image code", IEEE Transactions on Communications, vol. 31, no. 4, (1983), pp. 532-540.

[33] R. Achanta, S. Hemami, F. Estrada, and S. S, usstrunk. "Frequency-tuned salient region detection", In CVPR, (2009), pp. 1597-1604. 


\section{Authors}

Chunyan Yu, She is a lecture in the institute of information and science technology at dalian maritime university. Her current research interests include medical image processing, machine learning, intelligence computing.

Weishizhang, He is a professor in the institute of information and science technology at dalian maritime university. His current research interests include image processing, software engineering, intelligence computing.

Chunli Wang, She is a professor in the institute of information and science technology at dalian maritime university. Her current research interests include image processing, intelligence computing. 